\title{
Brief suicide preventive intervention in newly diagnosed HIV-positive persons
}

\section{Govender RD¹, Schlebusch L², Esterhuizen T ${ }^{3}$}

${ }^{1}$ Department of Family Medicine, University of KwaZulu-Natal, Durban, South Africa ${ }^{2}$ Emeritus Professor of Behavioural Medicine, University of KwaZulu-Natal, Durban, South Africa

${ }^{3}$ Programme of BioResearch Ethics and Law, Department of Public Health Medicine, College of Health Sciences, University of KwaZulu-Natal, Durban, South Africa

\begin{abstract}
South African studies have found that country wide suicide rates are high and that people diagnosed with HIV/AIDS can have increased suicidal ideation and resultant suicide risk. In this study, we evaluated the effect of a brief psychosocial intervention on preventing suicide ideation after a positive HIV test result. Suicidal ideation was assessed by both groups of patients having to complete a suicide risk screening scale (Annexure 1). The study was conducted at a university-affiliated hospital in Durban, KwaZulu-Natal, South Africa. Consenting adult patients (age 18 years and older) recently diagnosed as being HIV-positive following voluntary HIV counselling and testing were enrolled in the study. Participants (N=126) were assigned to standard post-test counselling (SPTC). Thereafter, every alternate patient $(\mathrm{N}=64)$ was counselled using a brief suicide preventive intervention (BSPI). Patients were assessed at baseline, 72 hours later and 6 weeks after a positive HIV test result. The balance of 62 participants who received SPTC only were the control group, and compared with the BSPI group. Although both groups benefited from post-test counselling, results from the BSPI group demonstrated a clinically significant decrease in suicidal ideation over the time period studied. The results provide preliminary evidence on the efficacy of a BSPI for recently diagnosed vulnerable HIV-positive persons and the importance of educating such patients on suicideprevention strategies.
\end{abstract}

Keywords: Brief suicide risk screening; HIV

Received date: $2-12-2013$

Accepted date: $30-01-2014$

doi: http://dx.doi.org/10.4172/Psychiatry.1000112

\section{Background}

Worldwide, suicide rates have increased by $60 \%$ in the last few decades, with a projected global suicide mortality rate of about 1.5 million people per annum by $2020 .^{1}$ Similarly, suicide has been recognised as a significant public health concern in South Africa (SA) where the national suicide mortality rate has steadily increased over the past few decades from about $1 \%$ in the 1980's to approximately $11 \%$ or more currently of all unnatural deaths. ${ }^{2-4}$ Aside from the numerous complexities associated with suicide, the country faces the added burden of the HIV/AIDS pandemic with one of the largest seropositive populations globally and KwaZulu-Natal province (where the present study was done) bearing one of the highest proportions of this load. ${ }^{5}$

Suicidal ideation is defined as having the intent to commit suicide, wanting to take one's own life or thinking about committing suicide with or without actually making plans to do so. ${ }^{6}$ Although there is a paucity of suicide and HIV/AIDS research in Africa ${ }^{7}$, the studies done, have shown a high suicide risk in this population. ${ }^{6-10}$ As early as 1995, AIDS phobia was cited as a trigger in $17 \%$ of parasuicide cases among youth in SA. ${ }^{11}$ HIV-positive people are at risk for suicide ${ }^{2,6}$ and when first diagnosed with HIV, many individuals react with disbelief, anxiety and fear for what lies ahead. In a previous study, the most commonly endorsed statement by recently diagnosed HIV-positive persons was, "I can't imagine what my life would be like in 10 years". ${ }^{6}$ It is known that in many cases a positive HIV diagnosis can be linked to hopelessness and depression with the potential risk for suicidal ideation ${ }^{6,12-14}$, and that one of the most vulnerable periods for suicide risk is in the immediate post HIV-diagnosis period. ${ }^{9-10,14}$ Depression and anxiety are common in people living with HIV/AIDS. ${ }^{6,12,13}$ Not only have studies shown that HIV infection is associated with a greater risk for such disorders,${ }^{13}$ but untreated depression increases the risk of suicidal behaviour and or suicidal ideation..$^{6,13}$

Suicide prevention is defined as any self-injury prevention or health-promotion strategy that is generally or specifically aimed at reducing the incidence and prevalence of suicidal behaviour. ${ }^{15}$ Amongst others, suicide intervention includes: early recognition and assessment of risk; immediate response to suicide risk; resource referrals; and follow-up management and treatment of at-risk individuals. ${ }^{16}$ Although various intervention approaches have been described to prevent suicidal behaviour, ${ }^{16} \mathrm{a}$ complete and comprehensive preventive model is the universal/selective/indicated (USI) model, which targets the general population, vulnerable populations and persons at high risk for suicide. Universal preventive interventions are directed at entire populations; selective interventions are directed at individuals who are at greater risk for suicidal behaviour; and indicated preventions target individuals who have already begun to display self-destructive behaviour. ${ }^{16}$ In addition, a systematic review of suicide-prevention 
strategies has suggested five key areas for intervention, viz.:(i) education and awareness; (ii) screening for atrisk persons; (iii) treatment of psychiatric disorders; (iv) restricting access to lethal means; and (v) media reporting of suicide. ${ }^{17}$

There is growing evidence that suicide is increasing in the context of HIV/AIDS with HIV-positive persons having a 3 times higher prevalence than the general population despite the introduction of Antiretroviral therapy (ART). ${ }^{18}$ Extensive research ${ }^{19}$ as part of the World Health Organization's worldwide initiative for the prevention of suicide across five continents, found that brief intervention can be an important component of suicide prevention programmes and confirmed that indicated suicide prevention strategies should complement universal and selective suicide prevention strategies. Given this, the aim of the present study was to assess the effect of a brief suicide preventive intervention on suicidal ideation in HIV-positive persons immediately post-test, within 72 hours and 6 weeks later.

\section{Patients and Methods}

The study was conducted at a university-affiliated hospital in Durban, South Africa. The study site was at a district level health facility in the KwaZulu-Natal province which has one of the highest local provincial HIV prevalence rates.5 Patients attending the hospital clinic for voluntary counselling and testing (VCT) for the HIV were informed about the study. Those meeting the inclusion criteria (age 18 years and older and testing seropositive, i.e. HIV Stage 1 and 2) were approached for voluntary participation $(N=126)$. Participants received standard post-test counselling (SPTC) administered by the resident hospital VCT nurse counselor and conducted individually. Thereafter, every alternate patient was counselled utilizing a brief suicide preventive intervention (BSPI) administered by a trained BSPI counsellor. Both the SPTC and the BSPI were done in patients' preferred language of either English or isiZulu (the predominant languages spoken). The counsellor was certified-trained in HIV pre-post test counselling. Additionlly demographic data was collected (age, gender, educational status, marital status, ethnicity and religion). The BSPI participants constituted the intervention group $(N=64)$ and were then compared with the balance of patients $(N=62)$ who received SPTC only (the control group). Because some patients were lost on follow-up and not all patients provided relevant data, patient samples varied as depicted in the tables.

After counselling, participants in both groups were asked to complete a self-administered suicide risk screening scale (SRSS) ${ }^{20}$ (Annexure 1) at baseline, after 72 hours and 6 weeks later. The 14-item SRSS was developed previously from a shortened version of the Beck Hopelessness Scale (BHS) and the Beck Depression Inventory (BDI). ${ }^{20}$ The scale was tested for validity, internal consistency and a receiver operating characteristic (ROC) analysis was performed for sensitivity and specificity for suicidal ideation (positive at a score of $\geq 4$ ). The area under the ROC curve (AUC) was regarded as the probability of correct prediction. The AUC was $0.730(p<0.001 ; 95 \%$ confidence interval (CI) $0.64-0.81)$ at baseline and $0.776(p<0.001 ; 95 \%$ CI $0.68-$ $0.87) 3$ weeks thereafter. Accordingly, the SRSS score was considered to be a good predictor of suicidal ideation in the population studied. ${ }^{20}$ Hopelessness was also assessed using a pre-determined score of $\geq 5$ (items Vl-Vl1; Annexure 1) and direct suicidal risk using a score of $\geq 1$ (itemsV12-V14; Annexure 1). The SRSS was translated into isiZulu and back-translated to accommodate isiZulu speaking patients (one of the largest language groups served by the clinic) so as to avoid possible language bias. The translation was performed by a professional linguist and had been through rigorous review by the ethics committee.

In the model used for the SPTC group the main principles included:

1. Addressing the impact of disclosure of the HIV test result, allowing the participant to digest this information, and giving the participant time to explore his/her feelings and fears.

2. Considering that the participant may be emotionally 'shocked', and giving reassurance that he/she would not be abandoned by the team of healthcare professionals who have much to provide in terms of different treatment modalities.

3. Explaining to each participant that coming to terms with the result may take some time and that he/she can remain healthy by taking care of him-/herself, which includes following the recommended treatment plan and an appropriate diet, and seeing a doctor when necessary.

4. Addressing the need to understand the different modes of HIV transmission and how the virus can be transmitted to others, emphasising safe-sex practices and condom use, and explaining the natural history of the progression of the disease.

5. Explaining the intervention or treatment programmes available and changes in lifestyle, and emphasising that hospital social workers would be a part of the programme and would assist with social and support resources.

The BSPI included an extra one-hour individual therapy session that addressed additional psychosocial issues related to HIV-positivity at the time of presentation, and entailed identifying and helping to resolve interpersonal difficulties which may cause or exacerbate psychological distress. To render the BSPI effective and relevant to the target population, it was conducted according to a protocol that encompassed:

1. Feedback on research-based epidemiology and the risk for suicidal behaviour as indicative of psychological and/or social distress following VCT and a seropositive result.

2. Exploring potential suicide risk and protective factors and how patients should deal with such risk factors.

3. Expressing empathy and discussing the situation in light of the participant's personal circumstances, while maintaining objectivity and being non-judgemental.

4. Providing simple advice on how to live positively and encouraging personal responsibility to change behaviour, and encouraging self-efficacy and the participant's belief in his/her ability to make meaningful changes.

\section{Highlighting sociodemographic protective factors.}

6. Discouraging personalisation of psychosocial factors such as stigmatisation, fear of disclosure and discriminatory gender issues.

7. Openly discussing HIV/AIDS, including prevention and treatment, to help to de-stigmatise the disease, and discussing alternative coping mechanisms in case of suicidal ideation. 
8. To prevent participants from playing a passive role, the counsellor focussed on re-enforcing a positive participant mindset, discussing referral options where more intensive psychological/psychiatric treatment was required, encouraging the possibility of family therapy if the patient was agreeable increasing the patient's sense of personal value, advising the patient to seek help when difficulties arise, encouraging openness to exploring potential suicide risk factors, garnering support from social networking and relevant people, and developing a renewed sense of purpose in life.

\section{Ethics considerations}

The research protocol was approved by the University of KwaZulu-Natal Biomedical Research Ethics Committee. All enrolled patients provided written informed consent for participation. All patients considered to be at high risk for suicidal ideation were referred for appropriate psychiatric/ psychological treatment.

\section{Statistical analyses}

Strata software (version 12) was used for statistical analysis. Generalised linear modelling was used to categorise participants with suicidal ideation. Pearson's chi-square test was used to determine the statistical significance of differences between the control and intervention groups. McNemar's chi-square test was used for paired binary proportions.

\section{Results}

Table 1 depicts the assessment of suicidal ideation among participants following SPTC and BSPI at the 3 time-points (baseline, 72 hours and 6 weeks after a positive HIV test result). Although Pearson's chi-square tests showed no statistically significant outcome between the SPTC and BSPI at all 3 timepoints, the trend analysis for suicidal ideation from baseline, to 6 weeks (Table 2) showed a suicidal ideation incidence of $019.79 / 1000$ person days (95\% CI $14.31-25.28)$ in the SPTC group and 015.87/1 000 person days (95\% CI 11.07 - 20.67) in the BSPI group. In comparison with the control group, the crude incidence rate ratio for suicidal ideation among the intervention group was 0.80 (95\% CI 0.52 - 1.23). Therefore, although both groups benefitted from post-test counselling, the BSPI proved more protective against the possibility of suicidal ideation in the patients studied. In addition, there was a significant change from positive (suicidal ideation) to negative (no suicidal ideation) from baseline to 72 hours and from baseline to 6 weeks. The change from 72 hours to 6 weeks was not statistically significant. This suggests

Table 1: Suicidal ideation following SPTC and BSPI at 3 timepoints after a positive HIV test result

\begin{tabular}{|c|c|c|c|}
\hline \multirow{2}{*}{ Time-point } & \multicolumn{2}{|c|}{ Outcome (suicidal ideation $\geq 4$ ) } & \multirow{2}{*}{$p$-valuea } \\
\hline & Negative $\mathrm{n}(\%)$ & Positive $\mathrm{n}(\%)$ & \\
\hline Baseline & & & 0.275 \\
\hline Control $^{b}$ & $25(40.3)$ & $37(59.7)$ & \\
\hline BSPI & $32(50.0)$ & $32(50.0)$ & \\
\hline 72 hours & & & 0.610 \\
\hline Control $^{b}$ & $44(71.0)$ & $18(29.0)$ & \\
\hline BSPI & $48(75.0)$ & $16(25.0)$ & \\
\hline 6 weeks & & & 0.189 \\
\hline Controlb & $42(67.7)$ & $20(32.3)$ & \\
\hline BSPI & $50(78.1)$ & $14(21.9)$ & \\
\hline
\end{tabular}

\begin{tabular}{|c|c|c|c|}
\hline & BSPI & Controla & Total \\
\hline Cases $(n)$ & 42 & 50 & 92 \\
\hline Patient time & 2646 & 2526 & 5172 \\
\hline \multirow[t]{2}{*}{ Incident rate } & 0.015873 & 0.0197941 & 0.0177881 \\
\hline & \multicolumn{2}{|c|}{ Point estimate $^{b}$} & $95 \% \mathrm{Cl}$ \\
\hline Incident rate difference & \multicolumn{2}{|c|}{-0.0039211} & $\begin{array}{c}-0.0112113- \\
0.0033691\end{array}$ \\
\hline Incident rate ratio (exact) & \multicolumn{2}{|c|}{0.8019048} & $0.5191035-1.233345$ \\
\hline $\begin{array}{l}\text { Previous fraction explained } \\
\text { (exact) }\end{array}$ & \multicolumn{2}{|c|}{0.1980952} & $\begin{array}{l}-0.2333446- \\
0.4808965\end{array}$ \\
\hline Previous fraction for population & \multicolumn{2}{|c|}{0.1013457} & \\
\hline \multicolumn{4}{|c|}{$\begin{array}{l}\text { aSPTC only. } \\
\text { b(midp) } \operatorname{Pr}(k<=42)=0.1466 \text { (exact) } \\
\text { (midp) } 2^{*} \operatorname{Pr}(k<=42)=0.2932 \text { (exact) }\end{array}$} \\
\hline
\end{tabular}

\begin{tabular}{|c|c|c|}
\hline Group & $\begin{array}{l}\text { Number of valid } \\
\text { cases }(n)\end{array}$ & Exact Sig. (2-sided) \\
\hline Controlb $^{b}$ & 62 & 0.000 \\
\hline BSPI & 64 & 0.000 \\
\hline Total & 126 & 0.000 \\
\hline \multicolumn{3}{|c|}{$\begin{array}{l}\text { aMcNemar's chi-square test; Binomial distribution used. } \\
\text { bSPTC only. }\end{array}$} \\
\hline
\end{tabular}

that the maximum change from positive to negative took place in the first 72 hours after the BSPI. Table 3 depicts the corresponding statistical significance (using McNemar's chi-square test) of the effectiveness of the BSPI, from having suicidal ideation (positive) at baseline to having no thoughts of suicide (negative) 6 weeks later.

The first 11 items of the SRSS (Annexure I) measures features of hopelessness. Consistent with previous findings, 6 descriptive statistics for each of the SRSS statements revealed that the statement, "I can't imagine what my life would be like in ten years" (Annexure 1) had the highest affirmative score in both the control and intervention groups at all 3 time-points, although in the BSPI group there was a significant abnormal reduction in this view over the time period studied (Table 4).

\section{Discussion}

HIV/AIDS has been shown to be associated with a higher risk for suicide in certain patients. ${ }^{2,6-10}$ Brief interventions have consistently been found to be effective in different patient populations. ${ }^{19-21}$ In this study, the implementation of a brief suicide preventive intervention along with the standard post-test counselling decreased suicidal ideation in seropositive patients particularly within the first 72 hours. We can consider these first 72 hours as the 'golden hours of suicide prevention' in HIV-positive persons. The added advantage is that during this early period those patients that are lost to follow up over a longer time frame would still have the benefit of suicide intervention treatment. Within the South African context, this is an absolute advantage, given that so many HIV-positive persons are lost to follow up. More importantly, in South Africa, suicide risk assessment and interventions are limited by the shortages of adequately trained health care professionals, suicide risk screening and suicide preventive intervention guidelines. HIV counsellors 


\begin{tabular}{|c|c|c|c|c|c|c|}
\hline \multirow{2}{*}{ SRSS item } & \multicolumn{2}{|c|}{ Baseline } & \multicolumn{2}{|c|}{72 hours } & \multicolumn{2}{|c|}{6 weeks } \\
\hline & Control $^{\mathrm{b}}(\%)$ & BSPI (\%) & Control (\%) & BSPI (\%) & Control (\%) & BSPI (\%) \\
\hline V1 & 51.6 & 43.8 & 43.5 & 42.2 & 38.3 & 39.7 \\
\hline V2 & 83.9 & 78.1 & 62.9 & 56.3 & 63.3 & 55.6 \\
\hline V3 & 32.3 & 25.0 & 17.7 & 18.8 & 21.7 & 17.5 \\
\hline V4 & 35.5 & 32.8 & 21.3 & 20.3 & 13.3 & 11.1 \\
\hline V5 & 47.5 & 42.2 & 24.2 & 23.4 & 21.7 & 20.6 \\
\hline V6 & 50.0 & 40.6 & 19.4 & 14.1 & 18.3 & 19.0 \\
\hline V7 & 50.8 & 45.3 & 17.7 & 15.6 & 31.7 & 22.2 \\
\hline V8 & 36.1 & 31.3 & 17.7 & 12.5 & 18.3 & 14.3 \\
\hline V9 & 50.0 & 40.6 & 23.0 & 20.3 & 23.3 & 14.3 \\
\hline V10 & 38.7 & 37.5 & 16.1 & 14.1 & 23.3 & 12.7 \\
\hline V11 & 37.1 & 31.3 & 6.5 & 3.1 & 15.0 & 4.8 \\
\hline V12 & 3.3 & 3.1 & 4.8 & 3.1 & 5.0 & 1.6 \\
\hline V13 & 0.0 & 0.0 & 0.0 & 0.0 & 0.0 & 0.0 \\
\hline V14 & 11.5 & 10.9 & 1.6 & 1.6 & 0.0 & 0.0 \\
\hline
\end{tabular}

and primary care physicians who are responsible for preand post-test HIV counselling and psychosocial education can easily be task-shifted to screen and provide suicide interventions resulting in effective reduction of suicidal ideation at a reasonable cost and minimal training.

Although the BSPI was intended as an intervention to reduce suicidal ideation in recently diagnosed seropositive patients, its value to potentially prevent eventual suicidal behaviour at a later stage cannot be underestimated because of the various suicide risk phases HIV/AIDS patients may go through. ${ }^{2,6}$ Importantly, a multi-site research study using standardised methodology ${ }^{22}$ has shown that suicidal ideation can be construed as a sign of distress and that there is a strong cultural underpinning underlying suicidal behaviour. ${ }^{22}$ Our findings suggest that witin this context the BSPI assisted in decreasing hopelessness and psychosocial stress, thereby helping the patients to cope better and attenuate suicidal ideation. This is supported by other studies, ${ }^{23-26}$ which showed that people living with HIV/AIDS can develop enhanced coping skills if they have access to medical treatment and a strong and supportive social network.

Resilience in individuals facing adversity is considered to be attributed to a combination of personal and contextual resources that enable effective adjustment to challenges and life situations. ${ }^{23}$ This implies that with appropriate help, HIV-seropositive individuals can overcome the perception of adversity brought on by the infection, and in some patients it may be this resilience that serves as a protective factor or coping mechanism ${ }^{27}$ which can also be postulated to be one of the effects of the BSPI. Furthermore, studies have shown that the duration of psychosocial intervention can be relevant to its effectiveness. ${ }^{28,29}$ In the present study the maximum change in reducing suicidal ideation occurred within the first 72 hours following a positive HIV diagnosis, which confirms the value of early intervention to prevent suicidality in these patients.

\section{Study limitations}

Although the results of the present study provide valuable information on how to reduce suicidal ideation in newly diagnosed HIV-positive individuals, important limitations should be considered when interpreting the research findings. Firstly, the study's overall generalisability needs to be considered. The sample sizes were not large and the study was confined to the post-HIV-test period, with the biggest part of the study population being urban-based. Thus, the results should be interpreted with caution. Secondly, the participants should be followed up for a longer period than 6 weeks to determine the prolonged effectiveness of the intervention. This together with the relatively small sample size might have contributed to a lower statistical significance in this study, as suggested in a meta-analysis of studies on psychosocial interventions. ${ }^{28}$ Further research is required to gain greater clarity regarding the presence of suicidal ideation at the different stages of HIV infection and the effectiveness of suicide preventive intervention at these different stages within the context exposure to antiretroviral treatment.

\section{Conclusion}

In the present study, suicidal ideation was reduced in both the SPTC and BSPI groups, but to a greater degree in the latter, suggesting that although general counselling can have a positive psychological outcome, the BSPI was more effective in reducing suicidal ideation. We showed a decrease in the levels of suicidal ideation in newly diagnosed HIV-positive persons following appropriate counselling and BSPI. Thus the findings of our study support the value of such an approach in recently diagnosed HIVpositive persons in the primary care setting and especially for those presenting with either overt or covert signs of hopelessness, depression and suicidal ideation. All health care workers at HIV/AIDS clinics, but especially in poorly resourced countries, should be trained to increase their knowledge regarding suicide prevention and reduce suicidal ideation in vulnerable HIV-positive patients.

\section{Declaration}

The authors declare that this is original work and has not previously been published.

\section{Conflict of Interest}

None. 


\section{Acknowledgements}

The authors wish to thank the fieldworkers for their excellent work ethic and the collection of data for this research. We express sincere gratitude to Ms P Pillay for research assistance.

Financial acknowledgements: RDG was supported by a Doctoral Research Grant Award (2010) funded by the University of KwaZulu-Natal.

\section{References}

1. Bertolote JM, Fleischmann A. A global perspective on the magnitude of suicide mortality. In: D. Wasserman, \& C. Wasserman, eds, Oxford Textbook of Suicidology and Suicide Prevention. A Global Perspective. Oxford: Oxford University Press, 2009; 91-98.

2. Schlebusch L. Suicidal behaviour in South Africa. Pietermaritzburg: University of KwaZulu-Natal Press, 2005.

3. Flisher AJ, Parry CDH. Suicide in South Africa. Act Psychiat Scand 1994; 90: 348-353.

4. Schlebusch L. Suicide prevention: a proposed national strategy for South Africa. Afr J Psych 2012;1 5: 436-440.

5. Actuarial Society of South Africa (ASSA). ASSA 2008 AIDS and Demographic Model (lite version 110207). Pretoria: ASSA, 2011. http://aids.actuarialsociety.org.za/ ASSA2008-Model-3480.htm (accessed 16 July 2013).

6. Govender RD, Schlebusch L. Hopelessness, depression and suicidal ideation in HIV-positive persons. S Afr J Pysch 2012; 18:16-21.

7. Kinyanda E, Hoskins S, Nakku J, Nawaz S, Patel V. The prevalence and characteristics of suicidality in HIV/ AIDS as seen in an African population in Entebbe district, Uganda. BMC Psychiat 2012; 12: 63.

8. Skinner D, Mfecane S. Stigma, discrimination and the implications for people living with HIV/AIDS in SA.J Soc Aspects of HIV/AIDS 2004; 1: 157-164.

9. Govender RD, Schlebusch L. Suicidal ideation in seropositive patients seen at a South African HIV voluntary counselling and testing clinic. Afr J Psychiat 2012; 15:9498.

10. Schlebusch L, Govender RD. Age, gender and suicidal ideation following voluntary HIV counselling and testing. Int J Environ Res Public Health 2012; 9: 521 1-530.

11. Mhlongo T, Peltzer K. Parasuicide among youth in a general hospital in South Africa. Curationis 1999; 22: 72-6.

12. Ciesla GR, Roberts JE. Meta-analysis of the relationship between HIV infection and risk for depressive disorders. Am J Psychiat 2001; 158: 725-730.

13. Olley BO, Seedat $S$, Nei DG, Stein DJ. Predictors of major depression in recently diagnosed patients with HIV/AIDS in South Africa. AIDS Patient Care STDs 2004; 18: 481-487.

14. Thom R. Common mental disorders in people living with HIV/AIDS. S Afr J HIV Med 2009; 10: 8-13.

15. Silverman MM, Felner RD. The place of suicide prevention in the spectrum of intervention: Definitions of critical terms and constructs. Suicide Life Threat Behav 1995; 25 . 70-81.

16. Nordentoft M. Crucial elements in suicide prevention strategies. Prog Neuropsychopharmacol Biol Psychiat 2011; 35: 848-853.

17. Mann JJ, Apter A, Bertolote J, Beautrais A, Currier D, et al. Suicide prevention strategies: A systematic review. JAMA 2005; 294: 2064-2074.

18. Carrico AW. Elevated suicide rate among HIV positive persons despite benefits of antiretroviral therapy: Implications for a stress and coping model of suicide.Am J Psychiatry 2010; 167:117-119.[http://dx.doi.org/10.1176/ appi.ajp.2009.09111565]

19. Fleischmann A, Bertolote JM, Wasserman D, De Leo D, Bolhari J, et al. Effectiveness of brief intervention and contact for suicide attempters: a randomized controlled trial in five countries. Bull World Health Organ 2008; 86 . 703-709.

20. Bertolote JM, Govender RD, Schlebusch L. A suicide risk screening scale for HIV-infected persons in the immediate post-diagnosis period. S Afr J HIV Med 2013; 14: 58-63.

21. Bien TH, Miller WR, Tonigan JS. Brief interventions for alcohol problems: A review. Addiction 1993; 88:315-36. PMID:8461850 doi:10.1111/j.1360-0443.1993.tb00820

22. Fleischmann $A$, de Leo D, Wasserman D. Suicidal thoughts, suicide plans and attempts in the general population on different continents. In: D. Wasserman, C. Wasserman, eds, Oxford Textbook of Suicidology and Suicide Prevention. A Global Perspective. Oxford: Oxford University Press, 2009; 99-104.

23. Carvalho FT, Morais NA, Koller SH, Piccinini CA. Protective factors and resilience in people living with HIV/AIDS. Cad Saude Publica 2007; 23: 2023-33.

24. Zhang $Y$, Zhang $X$, Aleong TH, et al. Impact of HIV/AIDS on Social Relationships in Rural China. The Open AIDS Journal 2011; 5: 67-73.

25. Ncama BP, McInerney PA, Bhengu BR, Corless IB, Wantland $D J$, Nicholas $P K$, et al. Social support and medication adherence in HIV disease in KwaZulu-Natal, South Africa. Int J Nurs Stud 2008; 45(12):1757-1563.

26. Waddell EN, Messeri PA. Social support, disclosure, and use of antiretroviral therapy. AIDS Behav 2006; 10:263-272.

27. Seery MD, Leo RJ, Lupien SP, Kondrak CL, Almonte JL. An Upside to adversity? Moderate cumulative lifetime adversity is associated with resilient responses in the face of controlled stressors. Psychological Science. 2013.

28. Crawford MJ, Thomas O, Khan N.Psychosocial interventions following self-harm. Systematic review of their efficacy in preventing suicide. Br J Psych 2007; 190: 11-17.

29. Rehse B, Pukrop R. Effects of psychosocial interventions on quality of life in adult cancer patients: meta analysis of 37 published controlled outcome studies. Patient Education and Counseling 50:2003; 179-186. 